\title{
Chlorothalonil induces metabolic syndrome in mice by regulating host gut microbiota and bile acids metabolism via FXR pathways
}

\section{Zhiyuan Meng}

Yangzhou University

\section{Sen Yan}

China Agricultural University

Wei Sun

China Agricultural University

Jin Yan

China Agricultural University

Miaomiao Teng

Chinese Research Academy of Environmental Sciences

Ming Jia

China Agricultural University

Sinuo Tian

China Agricultural University

Ruisheng Li

China Agricultural University

Shiran Huang

China Agricultural University

\section{Yu Wang}

China Agricultural University

\section{Xiaojun Chen}

Yangzhou University

\section{Zhiqiang Zhou}

China Agricultural University

WenTao Zhu ( $\nabla$ wentaozhu@cau.edu.cn )

China Agricultural University https://orcid.org/0000-0001-6371-7998

\section{Research}

Keywords: Pesticides, Metabolic syndrome, Gut microbiota, Bile acids metabolism, FXR signal pathways 
Posted Date: October 29th, 2021

DOI: https://doi.org/10.21203/rs.3.rs-1000136/v1

License: (c) (i) This work is licensed under a Creative Commons Attribution 4.0 International License. Read Full License 


\section{Abstract}

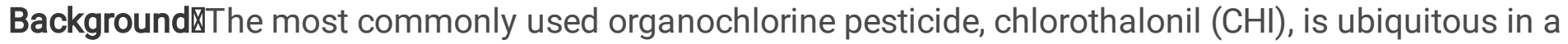
natural environment and poses many adverse effects to organisms. Unfortunately, the toxicity mechanisms of $\mathrm{CHI}$ have not been clarified yet.

Results: This study found that the low-dose $\mathrm{CHI}$ based on acceptable daily intake (ADI) level could induce metabolic syndrome (MetS) in mice, including obesity, hepatic steatosis, dyslipidemia, and insulin resistance. In addition, exposure to low-dose $\mathrm{CHI}$ could induce an imbalance in the gut microbiota of mice, resulting in a significant increase in the ratio of Firmicutes to Bacteroidetes. Furthermore, the results of the antibiotic treatment and gut microbiota transplantation experiments showed that the low-dose $\mathrm{CHI}$ could induce MetS in mice in a gut microbiota-dependent manner. Based on the results of targeted metabolomics and gene expression analysis, the low-dose $\mathrm{CHI}$ could disturb the serum metabolism of bile acids (BAs) in mice, causing the inhibition of the signal response of BAs receptor farnesol $\mathrm{X}$ receptor (FXR) and leading to glycolipid metabolism disorders in liver tissue and epididymal white adipose tissue (epiWAT) of mice. The administration of FXR agonist GW4064 and CDCA could significantly improve the low-dose CHI-induced MetS in mice.

Conclusions: In conclusion, the low-dose $\mathrm{CHI}$ was found to induce MetS in mice by regulating the gut microbiota and BAs metabolism via the FXR signaling pathway. This study provides evidence linking the gut microbiota and pesticides exposure with the progression of MetS, demonstrating the key role of gut microbiota in the toxic effects of pesticides.

\section{Background}

In recent years, metabolic syndrome (MetS) has become an important public health issue worldwide [1]. As a multi-gene and multi-organ disease, MetS characterized by insulin resistance, obesity, dyslipidemia, and hepatic steatosis [2]. In the United States, it affects at least $30 \%$ of adults and has been reported to be associated with the increased incidence of type 2 diabetes, atherosclerosis, and coronary heart disease [3]. The occurrence of MetS is mainly affected by genetic and environmental factors. As an important environmental pollutant, exposure to pesticides causes MetS in the host. It has also been confirmed that exposure to pesticides, such as p,p'-DDE, chlorpyrifos, and imidacloprid, could induce dyslipidemia, insulin resistance, or obesity [4-7]. In addition, several epidemiological studies have shown clear correlations between the levels of organochlorine pesticides in the human body and the incidence of MetS $[8,9]$. As one of the most important organochlorine pesticides worldwide, chlorothalonil $(\mathrm{CHI})$ is used in agricultural production and effectively prevents the fungal diseases of many crops. Due to a large number of its applications, $\mathrm{CHI}$ is widely present in many natural environments, such as water, air, and soil [10]. In addition, it has also been detected in cabbage, tomato, and other vegetables, and its residual concentration even exceeds its maximum residue limit (MRL) $[11,12]$. Therefore, the effects of $\mathrm{CHI}$ on organisms' health have received more attention. Many previous studies have demonstrated the adverse effects of $\mathrm{CHI}$ on organisms [13-19]. It could not only inhibit ovarian development through endocrine 
disruption but could also impair spermatogenesis through epigenetic pathways in mice $[17,20]$. In addition, a recent study showed that long-term exposure to $\mathrm{CHI}$ led to developing symptoms related to MetS, including liver damage and glycolipid metabolism disorders in mice [21].

There is a dense and diverse microbial community in the intestine of mammals, known as gut microbiota [22]. In the human gut, there are about $10^{14}$ bacterial cells, which are 10 times the number of human cells. In addition, the gut microbiota has more than 5 million functional genes, which are related to many basic physiological functions, including metabolism and nutritional homeostasis, immune system maturation, and nervous system activity of the host. The imbalance of gut microbiota has been reported to be related to many human diseases, such as MetS, colitis, type 2 diabetes mellitus, etc. [2325]. Importantly, the regulation of host physiological functions by gut microbiota has been demonstrated to be closely related to the metabolites of gut microbiota [26, 27]. Bile acids (BAs), an important class of microbially-produced metabolites, can regulate several host processes by activating the nuclear receptors in the intestine, liver, and peripheral tissues [28]. As one of the most widely studied bile acid receptors, the Farnesol $X$ receptor (FXR) is a ligand-dependent transcription factor, which is widely distributed in organs rich in BAs [29]. Previous studies have shown that FXR plays an important role in the maintenance of energy homeostasis and regulation of glycolipid metabolism in the host [30,31]. The activation of FXR can effectively inhibit dyslipidemia and prevent liver steatosis in mice [32,33]. Moreover, the activation of FXR by BAs regulates glucose metabolism by improving insulin sensitivity and repressing hepatic gluconeogenesis $[28,29]$. Previous studies have demonstrated that the gut microbiota could cause dietinduced obesity through FXR signaling $[32,34,35]$. Furthermore, the microbiota induced the inflammation of adipose tissue and increased the expression levels of genes responsible for fatty acid uptake in an FXR-dependent manner [34]. In particular, several studies have reported that pesticide exposure can disrupt the gut microbiota of the host $[36,37]$. Among them, few studies have suggested that the gut microbiota plays an important role in the pesticides' effects on host health $[4,38]$. Importantly, previous studies have also shown that $\mathrm{CHI}$ exposure can lead to an imbalance of gut microbiota [21]. Based on previous studies, it was hypothesized that $\mathrm{CHI}$ could induce MetS-related symptoms through the BAs-FXR signaling pathway mediated by gut microbiota.

Furthermore, it is worth mentioning that most of the previous studies have focused on the toxic effects of exposure of organisms to high-dose pesticides $[13,18,19,21,39]$. The effects of low-dose pesticides on host health have been overlooked, while they might be capable of altering the gut microbiota with lasting consequences on hosts. Therefore, based on the acceptable daily intake (ADI) level, this study aimed at investigating the effects of $\mathrm{CHI}$ exposure on the phenotypes associated with MetS, the composition of gut microbiota, metabolic profiles of BAs, and expression of glycolipid metabolism-associated genes in mice. The underlying mechanism of low-dose chlorothalonil exposure-induced MetS in mice was elucidated using antibiotics treatment, gut microbiota transplantation, and FXR agonist treatment. The results indicated that the low-dose $\mathrm{CHI}$ exposure could induce MetS in mice by the regulation of gut microbiota and BAs via FXR signaling pathways. This study provided new insights into the pesticideinduced host health effects, demonstrated the role of gut microbiota in pesticide-induced host health 
effects, and provided valuable information for a comprehensive understanding of the health risks associated with pesticides and their mechanisms.

\section{Results}

\section{Low-dose CHI-induced MetS in mice}

After 12 weeks of exposure to $\mathrm{CHI}$, the body weights of the 18 week-old ICR mice increased significantly as compared to the Ctrl group. Eventually, the bodyweight of ICR mice also increased significantly from 6 weeks to 18 weeks (Fig. 1B). In addition, the weights of liver tissues and epiWATs also significantly increased after $\mathrm{CHI}$ exposure (Fig. $1 \mathrm{C}$ and D). Meanwhile, the ratios of liver weight and epiWAT weight to body weight were significantly changed in the $\mathrm{CHI}$ groups as compared to the Ctrl group (Fig. $1 \mathrm{C}$ and D). In particular, there were no significant differences in food and water intake between the two treatment groups (Fig. S2 A and B), indicating that the effects of $\mathrm{CHI}$ on mice, increasing their body weight, were not due to the increased food consumption. Furthermore, $\mathrm{CHI}$ significantly increased the contents of TC and LDL-C and significantly decreased that of TG in the serum of ICR mice, while that of HDL-C was not observed to change in the $\mathrm{CHI}$ group (Fig. 1E and F). Similarly, the content of glucose and insulin in the serum of ICR mice increased significantly with the $\mathrm{CHI}$ exposure. Importantly, exposure to $\mathrm{CHI}$ caused a significant increase in insulin resistance index (HOMA-IR) of ICR mice (Fig. 1G). Subsequently, the intrinsic effects of $\mathrm{CHI}$ on the liver tissue and epiWAT of ICR mice were further evaluated. The histopathological analysis showed that the $\mathrm{CHI}$ promoted lipids accumulation in liver tissues and caused liver damage (Fig. 1H). This was mainly due to the significant increase in the ALT activity in serum and content of TC contents in mice liver (Fig. $1 \mathrm{I}$ and $1 \mathrm{~J}$ ). In addition, the $\mathrm{CHI}$ also promoted lipids accumulation in the epiWAT tissue of ICR mice (Fig. $1 \mathrm{H})$. The average size of the epiWAT cells markedly increased in the $\mathrm{CHI}$ group as compared to $\mathrm{Ctrl}$ group (Fig. 1K). Meanwhile, the mRNA expression levels of IL22, TLR4, TNF- $a, I L 6, M C P 1, I L-1 \beta$, and IFN- $y$, as inflammatory marker genes in liver tissue and epiWAT, were quantified. The results showed that $\mathrm{CHI}$ significantly increased the mRNA expression levels of IL22, TLR4, TNF-a, IL6, and MCP1 genes in the liver tissue and epiWAT (Fig. S3). These results also indicated that $\mathrm{CHI}$ could cause obesity, hepatic steatosis, dyslipidemia, and insulin resistance in ICR mice. Furthermore, obesity, hepatic steatosis, dyslipidemia, and insulin resistance in C57BL/ 6 mice after $\mathrm{CHI}$ exposure were also observed (Fig. S1, Fig. S2 and Fig. S3). In short, these results implied that the lowdose exposure to $\mathrm{CHI}$ could induce MetS in mice.

\section{Low-dose $\mathrm{CHI}$ disrupted gut microbiota of mice}

The emerging exploration of the association between pesticides and the host's gut microbiota has completely changed the traditional understanding of pesticide-induced health effects of the host [36, 37]. Several studies have proved the close association between gut microbiota and MetS [40-42]. The imbalance of gut microbiota has been considered an important factor, inducing MetS in the host. Therefore, in this study, the effects of $\mathrm{CHI}$ on the gut microbiota of mice were evaluated using $16 S \mathrm{SRNA}$ gene sequencing. The principal component analysis (PCA), based on OTUs, showed that the gut 
microbiota of ICR mice altered with $\mathrm{CHI}$ exposure. PC1 and PC2 showed $23 \%$ and $19.5 \%$ variations, respectively. Differences in the gut microbiota between $\mathrm{CHI}$ and Ctrl groups Ewere observed (Fig. 2A). This indicated that $\mathrm{CHI}$ exposure induced remarkable changes in the overall structure and composition of gut microbiota in ICR mice. Furthermore, as a-diversity indicators of gut microbiota, the Simpson, Shannon, Chao1, and Observed_species indices did not change significantly in the ICR mice after $\mathrm{CHI}$ exposure (Fig. 2B). Subsequently, changes in the compositions of gut microbiota between the $\mathrm{CHI}$ and $\mathrm{Ctrl}$ groups were analyzed. The results showed that there were significant differences in the relative abundances of gut microbiota in ICR mice between the $\mathrm{CHI}$ and $\mathrm{Ctrl}$ groups (Fig. $2 \mathrm{C}$ and F). At the phylum level, exposure to $\mathrm{CHI}$ resulted in a significant increase and decrease in the relative abundances of Firmicutes and Bacteroidetes, respectively (Fig. 2D). Meanwhile, the ratio of Firmicutes to Bacteroidetes in the $\mathrm{CHI}$ group also increased significantly (Fig. 2E). At the genus level, the relative abundance of Clostridiales significantly increased, while that of S24-7, Lachnospiraceae, and Bacteroides significantly decreased in the $\mathrm{CHI}$ group as compared to the Ctrl group (Fig. 2G). In addition, the OTUs that significantly altered between the $\mathrm{CHI}$ and Ctrl groups also showed imbalances in the gut microbiota of ICR mice (Fig. $2 \mathrm{H})$. Similarly, $\mathrm{CHI}$ also caused significant changes in the gut microbiota of C57BL/6 mice (Fig. S4). The analysis of gut microbiota in $\mathrm{C} 57 \mathrm{BL} / 6$ mice showed that $\mathrm{CHI}$ altered the relative abundances of Firmicutes and Bacteroidetes and resulted in a significant change in the ratio of Firmicutes to Bacteroidetes at the phylum level (Fig. S4C-E). Moreover, at the genus level, the relative abundances of Clostridiales and S24-7 also changed significantly after CHI exposure (SI Appendix, Fig. S4 F and G). Collectively, these results demonstrated substantial alterations in the gut microbiota of mice exposed to a low dose of $\mathrm{CHI}$.

\section{Low-dose $\mathrm{CHI}$ altered BAs metabolic profiles of mice}

The effects of gut microbiota on a host's health are usually closely related to the metabolic axis of host gut microbiota $[27,43]$. The above results showed that, at the genus level, the low-dose $\mathrm{CHI}$ exposure caused significant changes in the relative abundances of Clostridiales, S24-7, and Bacteroides. Previous studies have also shown that Clostridiales, S24-7, and Bacteroides were closely related to the host's BAs metabolism $[28,29]$. In order to further explore the role of gut microbiota in low-dose CHI-induced MetS in mice, the contents of 41 BAs in serum were determined using UHPLC-MS/MS (Table S1). The typical chromatograms of 41 BAs are provided in Fig. S5. The lowest limit of detection (LLOD) was $0.24-1.95$ $\mathrm{nmol} / \mathrm{L}$ and the lowest limit of quantification (LLOQs) was $0.49-3.91 \mathrm{nmol} / \mathrm{L}$. The linear regression coefficients were all above 0.9733 . The quantitative results of $41 \mathrm{BAs}$ in the serum samples of each treatment group are listed in Tables S3 and S4. For ICR mice, the PCA analysis showed a significant difference between the $\mathrm{CHI}$ and $\mathrm{Ctrl}$ groups (Fig. $3 \mathrm{~A}$ ). In addition, as compared to the control group, the total BA contents in the serum of the $\mathrm{CHI}$ treatment group significantly increased (Fig. 3B). Additionally, the content of primary and conjugated BAs increased significantly (Fig. $3 \mathrm{C}$ and $\mathrm{F}$ ). In the $\mathrm{Ctrl}$ and $\mathrm{CHI}$ groups, a total of 18 BAs were detected (Fig. 3G). Among them, exposure to $\mathrm{CHI}$ caused significant changes in the content of $11 \mathrm{BAs}(\mathrm{Fig} .3 \mathrm{H})$. Specifically, the contents of $5 \mathrm{BAs}$, including CDCA, taurodeoxycholic acid (TDCA), cholic acid (CA), lithocholic acid (LCA), and deoxycholic acid (DCA), in the serum of $\mathrm{CHI}$ group significantly decreased (Fig. 3I). In addition, the contents of 6 BAs, including taurine- 
a-murocholic acid (T-a-MCA), taurine- $\beta$-murocholic acid (T- $\beta-M C A)$, taurolithocholic acid (TLCA), taurochenodeoxycholic acid (TCDCA), taurocholic acid (TCA), and ursodeoxycholic acid (UDCA), in the serum of the $\mathrm{CHI}$ group significantly increased (Fig. 3I). Consistently, the low-dose $\mathrm{CHI}$ exposure also caused significant changes to the BAs metabolic profiles in the serum of C57BL/ 6 mice (Fig. S6). Similar to the ICR mice, the contents of 3 BAs, including T-a-MCA, T- $\beta-M C A$, and UDCA, increased significantly, while that of 5 BAs, including CDCA, TDCA, CA, LCA, and DCA, decreased significantly in the serum of C57BL/ 6 mice after $\mathrm{CHI}$ exposure (Fig. S6I). In short, the low-dose $\mathrm{CHI}$ disrupts the BAs metabolism in mice.

\section{Low-dose $\mathrm{CHI}$ induced MetS in mice in a microbiota-dependent manner}

In order to further explore whether the low-dose $\mathrm{CHI}$ exposure-induced MetS in mice was dependent on the presence of gut microbiota, the ICR mice were treated with $\mathrm{CHI}$ and a mixture of antibiotics, containing vancomycin, neomycin, metronidazole, gentamicin, and ampicillin (Fig. 4A). Consistent with the above findings, the $\mathrm{CHI}$ exposure induced obesity, hepatic steatosis, dyslipidemia, and insulin resistance in the ICR mice as compared to Ctrl group. However, when the gut microbiota of mice was inhibited by antibiotics, the symptoms of MetS in the CHI-induced ICR mice showed significant improvement. Specifically, the body weight and body weight gain of ICR mice in the $\mathrm{CHI}+\mathrm{ABX}$ treatment group decreased significantly as compared to the $\mathrm{CHI}$ group (Fig. 4B). Consistently, the liver weight, epiWAT weight, and tissue coefficients were significantly reduced after $\mathrm{CHI}+\mathrm{ABX}$ exposure (Fig. $4 \mathrm{C}$ and D). In addition, the contents of TG, TC, and LDL-C in the serum of ICR mice after CHI+ABX exposure also decreased significantly (Fig. 4E and F). As compared to the $\mathrm{CHI}$ group, the serum glucose level, insulin level, and HOMA-IR index of the mice in $\mathrm{CHI}+\mathrm{ABX}$ group were significantly reduced. In addition, the histopathological analysis showed that the ABX treatment also improved the deposition of lipids in the liver tissue and epiWAT of mice (Fig. S7D). Meanwhile, the activities of ALT in serum and the contents of TC in liver were significantly down-regulated after exposure to CHI+ABX (Fig. S7A and B). Similarly, the average size of epiWAT cells in the mice treated with $\mathrm{CHI}+\mathrm{ABX}$ was significantly reduced (Fig. S7C). In summary, these results indicated that the low-dose $\mathrm{CHI}$ induced MetS in mice in a gut microbiotadependent manner.

\section{Low-dose CHI-induced MetS in mice were transferable through gut microbiota}

In order to further illustrate that gut microbiota mediated the occurrence of low-dose $\mathrm{CHI}$-induced MetS in mice, the cecal contents of low-dose $\mathrm{CHI}$-treated mice were transferred to the recipient mice, followed by the examination of MetS-related indicators (Fig. 5A). In order to verify the effectiveness of gut microbiota transplantation, 16S rRNA gene sequencing analysis was performed on the cecal contents of T-Ctrl and T$\mathrm{CHI}$ groups. The PCA analysis showed significant differences between the T-CHI and T-Ctrl groups (Fig. 5B). Consistently, as compared to the T-Ctrl group, the relative abundance of Firmicutes in the T-CHI group significantly increased, while that of Bacteroidetes decreased significantly (Fig. 5C). Meanwhile, the relative abundance ratio of Firmicutes to Bacteroidetes in the T-CHI group also increased significantly (Fig. 5D). In conclusion, the compositions of mice gut microbiota in the T-Ctrl and T-CHI 
groups were similar to that in the $\mathrm{Ctrl}$ and $\mathrm{CHI}$ groups, respectively (Fig. S8A-C). This indicated that the gut microbiota transplantation could effectively transplant the microbiota-associated phenotypes from the mice in $\mathrm{Ctrl}$ and $\mathrm{CHI}$ groups into the recipient mice. Subsequently, the MetS-related indicators in the recipient mice were analyzed. As compared to the T-Ctrl group, the bodyweight and body weight gain of mice in the T-CHI group increased significantly (Fig. 5E and Fig. S8D). Similarly, the weights of liver and epiWAT and its tissue coefficients also increased significantly in the T-CHI group (Fig. 5E and Fig. S9E and $\mathrm{F}$ ). In addition, the serum-related biochemical indicators in the T-CHI group also altered significantly. Specifically, the contents of TG and HDL-C significantly decreased, while those of LDL-C significantly increased in the T-CHI group (Fig. 5F). Meanwhile, the serum glucose levels, insulin levels, and HOMA-IR index of the mice in T-CHI group also increased significantly (Fig. 5G). The histopathological analysis showed that, as compared to the T-Ctrl group, the liver tissue and epiWAT of the mice in T-CHI group exhibited obvious lipid deposition (Fig. 5K). In comparison with the T-Ctrl group, the activities of ALT in serum and the contents TG of in liver in the T-CHI group altered significantly (Fig. $5 \mathrm{H}$ and $5 \mathrm{I}$ ). The size and area of epiWAT cells in the T-CHI group also increased significantly (Fig. 5J and Fig. S8K).

In addition, the metabolic profiles of serum BAs also altered significantly in the T-Ctrl and T-CHI groups (Tables S5 and Fig. S9A). In comparison with the T-Ctrl group, the contents total BAs in T-CHI group did not change significantly (Fig. S9B). However, the contents of secondary and unconjugated BAs were significantly down-regulated, while that of conjugated BAs were significantly up-regulated in the serum of T-CHI group mice (Fig. S9D-F). A total of 26 BAs were detected in the T-Ctrl and T-CHI groups (Fig. S9G). As compared to the T-Ctrl group, the contents of $13 \mathrm{BAs}$ in the serum of $\mathrm{T}-\mathrm{CHI}$ group changed significantly (Fig. S9H). Among them, the contents of TLCA, T- $\beta-M C A$, and TCDCA increased significantly $(P<0.05$, Fig. 2-10I). Moreover, the contents of $10 \mathrm{BAs}$, including a-murocholic acid (a-MCA), allocholic acid (ACA), CA, CDCA, 3-dehydrocholic acid (3-DHCA), LCA, DCA, taurodeoxycholic acid (TDCA), 23-deoxycholic acid medeoxycholic acid (23norDCA), and hyodeoxycholic acid (HDCA), decreased significantly (Fig. S9I). Together, these results demonstrated that the recipient mice recapitulated metabolic phenotypes as observed in their respective donor mice. The gut microbiota-mediated MetS and BAs metabolism disorders in mice were induced by low-dose $\mathrm{CHI}$ exposure.

\section{Low-dose CHI induced glycolipid metabolism disorders in mice by inhibiting BAs receptors FXR}

After exposure to low-dose $\mathrm{CHI}$, the metabolic profile of serum BAs metabolism in mice changed significantly. In particular, it was found that the major BAs, which could regulate the signaling pathway of an important nuclear BAs receptor FXR and included CDCA, CA, DCA, LCA, UDCA, TaMCA, and TBMCA, were all changed significantly [29]. This further prompted to explore the mRNA expression levels of $F x r$ and its main downstream genes, such as Shp, Srebp-1C, and Chrebp, in the liver and epiWAT tissues of ICR mice. The $\mathrm{CHI}$ exposure significantly inhibited the relative RNA expression of Fxr (Fig. 6A). In addition, the relative mRNA expression levels of Srebp-1c and Chrebp significantly increased. In particular, the $\mathrm{CHI}$ exposure also significantly reduced the relative mRNA expression levels of Shp in liver tissues of ICR mice (Fig. 6A). Similarly, the $\mathrm{CHI}$ exposure also significantly inhibited the relative mRNA expression of Fxrgene in liver tissue and epiWAT of C57BL/ 6 mice. Furthermore, the relative mRNA expression levels of 
Shp, Srebp-1c, and Chrebp were also significantly changed after $\mathrm{CHI}$ exposure in liver tissue and epiWAT of C57BL/6 mice (Fig. 6B). Importantly, the recipient mice after receiving the transplantation of gut microbiota also demonstrated significant changes in the mRNA expression levels of $F x r$ and other related genes. As compared to the T-Ctrl group, the mRNA relative expression of Fxr gene in the liver tissue and epiWAT of the T-CHI group was significantly down-regulated (Fig. S10A), while that of the Chrebp gene was significantly up-regulated (Fig. S10D). In addition, in the T-CHI group, the relative mRNA expression of Shp gene in the liver tissue was significantly reduced, while that of the Srebp-1c gene in the epiWAT was significantly increased (Fig. S10B and C). These results demonstrated that the low-dose $\mathrm{CHI}$ exposure could alter the mRNA expression levels of nuclear BAs receptor Fxr and its main downstream related genes.

As an important nuclear BAs receptor, Fxr regulates the expression of various genes, which are related to glycolipid metabolism. Furthermore, the mRNA expression levels of genes related to glycolipid metabolism in the liver tissue and epiWAT after $\mathrm{CHI}$ exposure were analyzed, which included fatty acid synthesis genes (Fasn, Acaca, Gpat1, Agpat1, Ppary, Mogat1, Dgat1 and Dgat2), fatty acid uptake and transport genes, (Cd36, Fabp1, Fabp2 and Fatp5), fatty acid oxidation genes (Cpt1a, Acot1, Acox1 and Ppara), lipoprotein secretion genes (Mttp and Apod), glycogen synthesis (Gys2), gluconeogenesis genes (Pepck and G6pase), glycolysis genes (Gck and Pk/r), and glucose transport gene (Glut2) (Fig. 6C). For the liver tissue of ICR mice, $\mathrm{CHI}$ exposure significantly increased the relative mRNA expression levels of Dgat1 and Fatp5 (Fig. 6D and E). As compared to the control group, the relative mRNA expression levels of Cpt1a and Ppara in the $\mathrm{CHI}$ group were significantly down-regulated (Fig. 6F). In addition, $\mathrm{CHI}$ exposure also significantly increased the relative mRNA expression levels of G6pase, Gck, and Glut2 (Fig. 6H). For the epiWAT of ICR mice, exposure to $\mathrm{CHI}$ significantly increased the relative mRNA expression levels of 6 fatty acid synthesis genes, including Fasn, Acaca, Agpat1, Ppary, Mogat1, and Dgat1 (Fig. 6D). Similarly, the relative mRNA expression levels of $\mathrm{Cd} 36$ and Fatp5 in the $\mathrm{CHI}$ group also significantly increased (Fig. $6 \mathrm{E})$. At the same time, exposure to $\mathrm{CHI}$ significantly reduced the relative mRNA expression levels of $\mathrm{Cpt1a}$, Acox1, and Ppara (Fig. 6F). In addition, $\mathrm{CHI}$ exposure also significantly increased the relative mRNA expression level of G6pase (Fig. 6H). In particular, $\mathrm{CHI}$ exposure could also promote the mRNA expression levels of fatty acid synthesis and uptake-related genes, inhibit the expression of fatty acid oxidationrelated genes, and activate the expression of gluconeogenesis -related genes in the liver tissue and epiWAT of C57BL/6 mice (Fig. S11). These results implied that CHI exposure could cause the disorders of glycolipid metabolism in the liver tissue and epiWAT of ICR and C57BL/6 mice. In conclusion, these results confirmed that the low-dose $\mathrm{CHI}$ exposure could induce glycolipid metabolism disorders in mice by inhibiting the FXR signaling pathway.

\section{FXR activators ameliorated the MetS of mice induced by low-dose $\mathrm{CHI}$}

In order to further determine the key role of BAs receptor FXR in the low-dose $\mathrm{CH}$-induced MetS, the ICR mice were treated with $\mathrm{CHI}$ and FXR activators, which included GW4064 and CDCA (Fig. 7A). The analysis of MetS-related indicators in each treatment group showed that both the FXR activators, GW4064 and CDCA, effectively ameliorated the low-dose $\mathrm{CHI}$-induced MetS in mice. In particular, as compared to the 
$\mathrm{CHI}$ group, the body weight and body weight gain of mice in the $\mathrm{CHI}+\mathrm{GW} 4064$ and $\mathrm{CHI}+\mathrm{CDCA}$ groups were significantly reduced (Fig. 7B and Fig. S12A). Similarly, in the CHI+GW4064 and CHI+CDCA groups, the weight of liver tissue and epiWAT and its tissue coefficients were also significantly reduced (Fig.7B and Fig. S12A). In addition, the GW4064 and CDCA activators also significantly reduced the serum glucose level, insulin level, and HOMA-IR index in mice (Fig. 7D). Meanwhile, the serum LDL-C contents in the $\mathrm{CHI}+\mathrm{GW} 4064$ group were significantly reduced as compared to the $\mathrm{CHI}$ group (Fig. 7C). Furthermore, the histopathological analysis showed no significant lipid deposition in the liver tissue and epiWAT of the mice in both the $\mathrm{CHI}+\mathrm{GW} 4064$ and $\mathrm{CHI}+\mathrm{CDCA}$ treatment groups as compared to the $\mathrm{CHI}$ group (Fig. 7G). As compared to the $\mathrm{CHI}$ group, the liver ALT activity and TC and TG contents in the CHI+GW4064 and CHI+CDCA groups were significantly reduced (Fig. 7E and Fig. S12E). Similarly, the size and area of epiWAT tissue cells in the $\mathrm{CHI}+\mathrm{GW} 4064$ and $\mathrm{CHI}+\mathrm{CDCA}$ groups were also significantly reduced (Fig. 7F and and Fig. S12F). These results indicated that both the FXR activators GW4064 and CDCA could effectively ameliorate the low-dose CHI-induced MetS in mice, such as obesity, hepatic steatosis, dyslipidemia, and insulin resistance.

\section{Discussion}

At present, MetS are developing into one of the most health-threatening diseases worldwide [3]. Previous studies have shown that many environmental pollutants, including pesticides, flame retardants, and plasticizers, might be unfavorable factors in inducing MetS [44-47]. Several epidemiological studies have shown a close association of organochlorine pesticides with host obesity or insulin resistance [8, 9]. In addition, a previous study has also found that exposure to organochlorine pesticide $\mathrm{CHI}$ can cause dyslipidemia and energy metabolism disorders in mice [21]. However, the adverse effects of $\mathrm{CHI}$ on developing MetS-related symptoms, such as obesity, lipid deposition, and insulin resistance in mice are still controversial.

This study confirmed that the low-dose $\mathrm{CHI}$ after 12 weeks of exposure could develop MetS-related symptoms, which included obesity, hepatic steatosis, dyslipidemia, and insulin resistance in the ICR and C57BL/ 6 mice. Based on previous studies conducted on the association of gut microbiota with MetS of host [40-42, 45], it was speculated that the low-dose $\mathrm{CHI}$-induced MetS might be related to the gut microbiota of mice. Furthermore, the composition of mice gut microbiota in each treatment group was determined using $16 S$ rRNA gene sequencing technology. The results showed that the low-dose $\mathrm{CHI}$ could cause the ecological imbalance of the gut microbiota of ICR mice and C57BL/6 mice. Consistently, as compared to $\mathrm{Ctrl}$ group, low-dose $\mathrm{CHI}$ significantly altered the relative abundances of Firmicutes and Bacteroidetes, resulting in a significant increase in the relative abundance ratio of Firmicutes to Bacteroidetes. In particular, both these phyla play key roles in obesity-related metabolic diseases mediated by host gut microbiota [48]. An increase in the ratio of Firmicutes to Bacteroidetes has been found in obese humans $[49,50]$. In addition, $\mathrm{CHI}$ also caused significant alterations in the relative abundances of Clostridiales, S24-7 and Bacteroides at genus level. Several studies have shown the close association of Clostridiales with host obesity [51]. However, previous reports have shown that S24-7 can promote the degradation of carbohydrates in host and its relative abundance has been reported to be 
significantly increased in mice fed a low-fat diet [52]. In addition, recent studies also indicated that Bacteroides alleviated obesity in mice and played an important role in human weight loss interventions $[53,54]$. These results implied that the low-dose $\mathrm{CHI}$ could induce MetS by changing the composition of mice's gut microbiota. In order to further clarify the role of gut microbiota in the low-dose $\mathrm{CHI}$-induced MetS in mice, antibiotic treatment and gut microbiota transplantation were carried out. The results confirmed that the antibiotic treatment could improve the low-dose $\mathrm{CHI}$-induced MetS. In addition, this MetS could be transferred to the recipient mice through the gut microbiota. These findings proved that the gut microbiota could mediate the low-dose $\mathrm{CHI}$-induced MetS in mice.

Several have shown that the gut microbiota could induce health effects by changing the host metabolic phenotype [55]. Many metabolites of gut microbiota act as signal molecules and substrates for the host's metabolic reactions, thereby regulating the multiple physiological processes of host [43]. In this study, significant changes in the relative abundances of Clostridiales, S24-7 and Bacteroides were found. Among them, the ability of Clostridiales to mediate the conversion of primary BAs to secondary BAs has been demonstrated $[56,57]$. In addition, Bacteroides can reduce the levels of T- $\beta$-MCA and TUDCA in the host, thereby improving the host MetS by activating the BAs-FXR signaling pathway in mice [54]. The effects of low-dose $\mathrm{CHI}$ exposure on the metabolic profiles of serum BAs in mice were studied using UPLC-MS/MS-based targeted metabolomics technology. The results indicated that the low-dose $\mathrm{CHI}$ exposure could significantly alter BAs metabolic profiles in the serum of ICR and C57BL/6 mice. Among them, the contents of CDCA and CA decreased significantly, while that of T- $\beta$-MCA and UDCA increased significantly. In particular, the recipient mice recapitulated BAs metabolism phenotype of their respective donor mice. As compared to the T-Ctrl group, the contents of CDCA and CA significantly decreased, while that of T- $\beta-M C A$ increased significantly in the T-CHI group. Currently, several studies have confirmed the interaction among MetS, gut microbiota, and BAs metabolism [58-60]. As effective ligands for BAs receptor FXR, the CDCA and CA can activate the FXR signaling pathway $[28,29,61]$. However, the T- $\beta-M C A$ and UDCA can significantly inhibit the FXR signaling pathway $[28,29,62]$. As the main BAs receptor, FXR is involved in the metabolism of host glycolipid and energy expenditure, especially maintaining the normal lipid metabolism and controlling inflammation [28, 29]. The overexpression of FXR in host liver tissue can prevent liver steatosis and regulate the levels of TG and BAs [63]. This study also showed that the low-dose $\mathrm{CHI}$ exposure could significantly reduce the mRNA expression of Fxrgene in liver tissue and epiWAT of ICR and C57BL/ 6 mice, leading to significantly alter the mRNA expression of Fxr downstream genes, including Shp, Srebp-1c, and Chrebp. The expression analysis of 24 genes involved in glycolipid metabolism in liver tissue and epiWAT of ICR and C57BL/6 mice showed that the low-dose CHI exposure promoted the fatty acid synthesis and uptake, inhibited the fatty acid oxidation, and activated gluconeogenesis. Subsequently, the key role of the BAs receptor FXR in CHI-induced MetS in mice was further confirmed through the FXR agonist treatment experiment. The results showed that the two FXR agonists GW4064 and CDCA could effectively improve CHI-induced obesity, hepatic steatosis, dyslipidemia, and insulin resistance in mice, respectively. In short, these results indicated that the disorders of glycolipid metabolism in liver tissue and epiWAT, mediated by the BAs receptor FXR, were the 
inherent reason for the low-dose $\mathrm{CHI}$-induction of MetS, which included obesity, hepatic steatosis, dyslipidemia, and insulin resistance in mice.

Overall, this study demonstrated that the low-dose $\mathrm{CHI}$ could induce MetS in mice by the regulation of gut microbiota and BAs via FXR signaling pathways (Fig. S13). This study revealed that gut microbiota could play a key role in the adverse effects of pesticides on organisms. This study also provided new insights into the mechanism of pesticide toxicity. In the future, the effects of pesticides on the host's gut microbiota should be evaluated more comprehensively and systematically.

\section{Conclusions}

In this study, it was found that the low-dose $\mathrm{CHI}$ could induce MetS in mice, which included obesity, hepatic steatosis, dyslipidemia, and insulin resistance. In addition, the low-dose $\mathrm{CHI}$ could also cause the dysbiosis of gut microbiota in mice. Antibiotic treatment and gut microbiota transplantation experiments suggested that the gut microbiota could mediate the $\mathrm{CHI}$-induced MetS in mice. In addition, the low-dose $\mathrm{CHI}$ also disturbed the metabolic profiles of BAs in mice. This further led to the significant inhibition of BAs receptor FXR signal, leading to the disturbance of glucose and lipid metabolism in the liver tissue and epiWAT of mice. In particular, the administration of FXR agonists effectively improved low-dose CHIinduced MetS in mice. In summary, the gut microbiota and BAs receptor FXR mediate the development of low-dose $\mathrm{CHI}$-induced MetS in mice.

\section{Methods}

\section{Reagents}

Chlorothalonil (CHI, purity 99.0\%), vancomycin (purity 98.5\%), neomycin (purity 99\%), metronidazole (purity 99.0\%), gentamicin (purity 97.5\%), ampicillin (purity 98\%), GW4064 (purity 98\%), and chenodeoxycholic acid (CDCA, purity 98.0\%) were purchased from Shanghai Aladdin (Shanghai, China). All the BAs standards were purchased from Sigma-Aldrich (MO, USA) and Steraloids (RI, USA).

\section{Animal Experiments}

The animal experiments were conducted according to the guidelines of the Institutional Animal Care and Use Committee of China Agricultural University (Approval no. AW52301202-2-1). ICR (CD-1) and C57BL/6 male mice were purchased from Beijing Vital River Laboratory Animal Technology Co., Ltd (Beijing, China). Then, the mice were maintained at room temperature $\left(22 \pm 2{ }^{\circ} \mathrm{C}\right)$ with a $12 / 12 \mathrm{~h} \mathrm{light} /$ dark cycle and ad libitum access to water and a normal diet based on AIN 93, which was obtained from Beijing Keao Xieli Feed Co., Ltd (Beijing, China).

\section{Animal protocol 1: Low-dose CHI exposure experiment}


Male ICR and C57BL/6 mice (age; 5 weeks) were randomly divided into two experimental treatment groups ( $\mathrm{n}=12$ in each group). The mice were orally administered with $\mathrm{CHI}$ deionized water solutions at a dose of $0.2 \mathrm{mg} / \mathrm{L}$ of $\mathrm{CHI}$ and named as $\mathrm{CHI}$ group. In this study, the dosages were set according to the ADI ( $0.02 \mathrm{mg} / \mathrm{kg} \mathrm{BW/day)} \mathrm{for} \mathrm{the} \mathrm{long-term} \mathrm{toxicity} \mathrm{of} \mathrm{mice.} \mathrm{The} \mathrm{control} \mathrm{group} \mathrm{(Ctrl)} \mathrm{was} \mathrm{administered}$ with deionized water only. After 12 weeks of treatment, the mice were euthanized. The serum samples, liver tissue, epididymal white adipose tissue (epiWAT), and cecal contents from each mouse were collected at the end of the experiment and stored at $-80^{\circ} \mathrm{C}$ for further analyses. The specific experimental design is presented in Fig. $1 \mathrm{~A}$ and Fig. S1A.

\section{Animal protocol 2: Antibiotic treatment experiment}

A total of 32 male ICR mice (age; 5 weeks) were randomly and equally assigned to 4 experimental treatment groups ( $n=8$ in each group). Among them, one group was administered with $0.2 \mathrm{mg} / \mathrm{L}$ of $\mathrm{CHI}$ containing deionized water solutions and named as $\mathrm{CHI}$ group. One group was administered with antibiotics-containing deionized water $(0.5 \mathrm{~g} / \mathrm{L}$ vancomycin, $1 \mathrm{~g} / \mathrm{L}$ neomycin sulfate, $1 \mathrm{~g} / \mathrm{L}$ metronidazole, and $1 \mathrm{~g} / \mathrm{L}$ ampicillin solutions) and named as $A B X$ group. One group was administered with the combination of $\mathrm{CHI}$ and antibiotics-containing deionized water $(0.2 \mathrm{mg} / \mathrm{L} \mathrm{CHI}, 0.5 \mathrm{~g} / \mathrm{L}$ vancomycin, $1 \mathrm{~g} / \mathrm{L}$ neomycin sulfate, $1 \mathrm{~g} / \mathrm{L}$ metronidazole, and $1 \mathrm{~g} / \mathrm{L}$ ampicillin solutions) and named as CHI+ABX group. One group was administered with deionized water only and named as Ctrl group. After 12 weeks of treatment, the mice were euthanized. The serum samples, liver tissue, epiWAT, and cecal contents from each mouse were collected at the end of the experiment and stored at $-80^{\circ} \mathrm{C}$ for further analyses. The specific experimental design is presented in Fig. 4A.

\section{Animal protocol 3: Gut microbiota transplantation experiment}

Preparation of gut microbiota suspension: The cecal contents of ICR mice from Animal protocol 1 were diluted with saline $(1: 9, \mathrm{w} / \mathrm{w})$ and homogenized for $1 \mathrm{~min}$ using a vortex to achieve a liquid slurry. The supernatants were collected by centrifugation at $500 \mathrm{~g}$ for $3 \mathrm{~min}$ to remove particulate matter and facilitate its administration and stored at $-80^{\circ} \mathrm{C}$.

Gut microbiota transplantation: The male ICR mice (age; 3 weeks) were orally administered with antibiotics-containing deionized water $(0.5 \mathrm{~g} / \mathrm{L}$ vancomycin, $1 \mathrm{~g} / \mathrm{L}$ neomycin sulfate, $1 \mathrm{~g} / \mathrm{L}$ metronidazole, and $1 \mathrm{~g} / \mathrm{L}$ ampicillin solutions) for 2 weeks. Then, these mice were randomly and equally assigned to 2 groups ( $n=6$ for each group), in which one group was re-colonized with the Ctrl group's microbiota and named as T-Ctrl group and another group was re-colonized with the $\mathrm{CHI}$ group's microbiota and named as $\mathrm{T}-\mathrm{CHI}$ group. Subsequently, the two groups were orally gavage-administered with $200 \mu \mathrm{L}$ of gut microbiota suspension for 1 week. In addition, both the groups were orally gavage-administered with $200 \mu \mathrm{L}$ of gut microbiota suspension 3 times a week for 2 weeks to consolidate the re-colonization of gut microbiota. After 18 weeks of normal diet, the mice were euthanized. The serum samples, liver tissue, epiWAT, and cecal contents from each mouse were collected at the end of the experiment and stored at $-80^{\circ} \mathrm{C}$ for further analyses. The specific experimental design is presented in Fig. 5A. 


\section{Animal protocol 4: FXR activators treatment experiment}

A total of 32 male ICR mice (age; 5 weeks) were randomly and equally assigned to 4 groups ( $\mathrm{n}=8$ for each group). Among them, one group was administered with $0.2 \mathrm{mg} / \mathrm{L}$ of $\mathrm{CHI}$-containing deionized water solution and named as $\mathrm{CHI}$ group. One group was administered with chlorothalonil and GW4064containing deionized water $(0.2 \mathrm{mg} / \mathrm{L} \mathrm{CHI}$ and $50 \mathrm{mg} / \mathrm{L} \mathrm{GW4064} \mathrm{solutions)} \mathrm{and} \mathrm{named} \mathrm{as} \mathrm{CHI+GW4064}$ group. One group was administered with $\mathrm{CHI}$ and CDCA-containing deionized water $(0.2 \mathrm{mg} / \mathrm{L} \mathrm{CHI}$ and $100 \mathrm{mg} / \mathrm{L}$ CDCA solutions and named as CHI+CDCA group. One group was administered with deionized water only and named as Ctrl group. After 12 weeks of treatment, the mice were euthanized. The serum samples, liver tissue, epiWAT, and cecal contents from each mouse were collected at the end of the experiment and stored at $-80^{\circ} \mathrm{C}$ for further analyses. The specific experimental design is presented in Fig. $7 \mathrm{~A}$.

\section{Food and water intake measurement}

The weight of food and volume of water provided to each group of mice were measured. After 24 hours, the remaining food weight and water volume were measured, and the food and water intakes per $24 \mathrm{~h}$ were calculated.

\section{Biochemical analysis and histopathology analysis}

The contents of serum triglyceride (TG), total cholesterol (T-Cho), low-density lipoprotein-cholesterol (LDL$\mathrm{C})$, and high-density lipoprotein-cholesterol (HDL-C) were measured using their respective assay kits. The activities of serum aspartate aminotransferase (AST) and alanine aminotransferase (ALT) were detected using their respective assay kits. The content of serum insulin was measured using commercial enzymelinked immunosorbent assay (ELISA) kits. In addition, the content of serum glucose was measured using the Beijing Yicheng blood glucose meter. The assay kits required for the above studies were purchased from Nanjing Jiancheng Bioengineering Institute (Nanjing, China). The liver tissue and epiWAT from all the treatment groups were randomly selected for histopathological analysis. The tissues were fixed in $10 \%$ neutral buffered formalin and embedded in paraffin for histological examination. The tissue sections were stained with hematoxylin-eosin (H\&E) and examined under light microscopy at 100x, 200x, or 400x magnification.

\section{Gut microbiota analysis}

According to the manufacturers' instructions, the QIAamp DNA stool mini kit (Qiagen, Germany) was used for the extraction of total bacterial metagenomic DNA from the cecal content samples of each group. The quantity and quality of extracted DNA were measured using a UV spectrophotometer and agarose gel electrophoresis, respectively. The PCR amplification of the bacterial $16 S$ rRNA gene hypervariable V3-V4 region was performed using forward primer 338F (5'-ACTCCTACGGGAGGCGC AG-CA-3') and reverse primer 806 R (5'-GGACTACHVGGG130 TWTCTAAT-3'). The PCR amplicons were purified using Agencourt AMPure Beads (Beckman Coulter, Indianapolis, IN) and quantified using the PicoGreen dsDNA Assay Kit 
(Invitrogen, Carlsbad, CA, USA). The sequencing was performed using the lllumina MiSeq platform with MiSeq Reagent Kit v3 at Shanghai Personal Biotechnology Co., Ltd (Shanghai, China). The Quantitative Insights into Microbial Ecology (QIIME, v1.8.0) pipeline was used to process the sequencing data.

\section{BAs targeted metabolomics analysis}

Metabolites Extraction: A total of $100 \mu \mathrm{L}$ of the serum sample was transferred to an Eppendorf tube. After the addition of $400 \mu \mathrm{L}$ of solvent extract (acetonitrile-methanol, 1:1, containing $0.1 \%$ formic acid and isotopically-labeled internal standard mixture), the samples were vortexed for $30 \mathrm{~s}$, sonicated for $10 \mathrm{~min}$ in an ice-water bath, incubation at $-40^{\circ} \mathrm{C}$ for $1 \mathrm{~h}$, and then centrifugation at $12000 \mathrm{rpm}$ and $4{ }^{\circ} \mathrm{C}$ for 15 min. The clear supernatants obtained were transferred to an auto-sampler vial for UHPLC-MS/MS analysis.

UHPLC-MS/MS Analysis: The UHPLC separation was carried out using a UHPLC System (Vanquish, Thermo Fisher Scientific), which was equipped with a Waters ACQUITY UPLC BEH C18 column (150* 2.1 $\mathrm{mm}, 1.7 \mu \mathrm{m}$, Waters). The Q Exactive HF-X mass spectrometer (Thermo Fisher Scientific) was used for assay development. The parallel reaction monitoring (PRM) parameters were optimized for each of the targeted analytes by injecting their respective standard solutions into the API source of the mass spectrometer. The detailed targeted bile acid metabolomics analysis methods are provided in supporting information SI.

\section{Quantitative RT-qPCR analysis}

Total RNA was extracted from the liver tissues and epiWAT of all the treatment groups using TRIzol reagent. Then, 1.5- $\mu \mathrm{g}$ RNA from each sample was reverse transcribed to form cDNA with random hexamer primers using a Fast Quant RT Kit (with gDNase) following the manufacturers' instructions. The TRIzol reagent and Fast Quant RT Kit were purchased from Tiangen Biochemical Technology (Beijing, China). RT-qPCR was performed with SuperReal PreMix Plus (SYBR Green) (Qiagen, China) using Bio-Rad CFX 96 PCR system (Bio-Rad, USA). The results of the target genes were normalized using the $2^{-\Delta \Delta C t}$ method. Gapdh gene was used as the internal control. The nucleotide sequences of mouse-specific primers were obtained from Sangon Biochemical Technology (Shanghai, China) and are listed in Table S1.

\section{Statistical analysis}

All the experimental values are expressed as mean \pm standard deviation (SD). The data of two treatment groups were analyzed using Student's $t$-test and those of more than two treatment groups were analyzed using one-way or two-way analysis of variance (ANOVA) with post hoc Tukey's test followed by significant difference tests using SPSS 19.0 (IBM, USA). All the results were considered statistically significant at $P<0.05$. Graphical illustrations were developed using GraphPad Prism version 6.0 (GraphPad Software, Inc., USA). The levels of significance indicated in the graphs are ${ }^{\star} P<0.05$ and $* \star P$ $<0.01$. 


\section{Declarations}

Acknowledgements

The authors thank the members from the laboratory animal center in China Agriculture University for their assistance of animal care.

\section{Funding}

This work was supportted from the National Key Research and Development Program of China (2016YFD0200202) and the 2115 Talent Development Program of China Agricultural University.

\section{Availability of data and materials}

All 16S rRNA gene sequencing reads data has been deposited to the National Center for Biotechnology Information (NCBI)'s Sequence Read Archive under accession number PRJNA772936.

\section{Authors' contributions}

ZM, SY, ZZ, XC and WZ designed the study. ZM, SY, WS, JY, MT, and MJ performed the experiments. ZM, $\mathrm{ST}, \mathrm{YW}$ and $\mathrm{RL}$ analyzed the data. $\mathrm{ZY}, \mathrm{SH}, \mathrm{XC}$ and $\mathrm{WZ}$ wrote the manuscript. All of the authors reviewed and approved the final manuscript.

\section{Ethics approval and consent to participate}

Institutional Animal Care and Use Committee of China Agricultural University.

\section{Consent for publication}

Not applicable.

\section{Competing interests}

The authors declare that they have no competing interests.

Author details

${ }^{1}$ Innovation Center of Pesticide Research, Department of Applied Chemistry, College of Science, China Agricultural University, Beijing 100193, China.

2 School of Horticulture and Plant Protection, Yangzhou University/Joint International Research Laboratory of Agriculture \& Agri-Product Safety (Yangzhou University), Jiangsu Yangzhou, 225009, China

${ }^{3}$ State Key Laboratory of Environmental Criteria and Risk Assessment, Chinese Research Academy of Environmental Sciences, Beijing 100012, China 


\section{References}

1. Boleti APD, Almeida JA, Migliolo L. Impact of the metabolic syndrome on the evolution of neurodegenerative diseases. Neural Regeneration Research. 2021;16(4):688-9; doi: 10.4103/16735374.295329.

2. Saklayen MG. The Global Epidemic of the Metabolic Syndrome. Current Hypertension Reports. 2018;20(2); doi: 10.1007/s11906-018-0812-z.

3. Ford ES, Li CY, Zhao GX. Prevalence and correlates of metabolic syndrome based on a harmonious definition among adults in the US. Journal of Diabetes. 2010;2(3):180-93; doi: 10.1111/j.17530407.2010.00078.x.

4. Liang YR, Liu DH, Zhan J, Luo M, Han JJ, Wang P, et al. New insight into the mechanism of POPinduced obesity: Evidence from DDE-altered microbiota. Chemosphere. 2020;244; doi:

10.1016/j.chemosphere.2019.125123.

5. Reygner J, Lichtenberger L, Elmhiri G, Dou S, Bahi-Jaber N, Rhazi L, et al. Inulin Supplementation Lowered the Metabolic Defects of Prolonged Exposure to Chlorpyrifos from Gestation to Young Adult Stage in Offspring Rats. Plos One. 2016;11(10); doi: 10.1371/journal.pone.0164614.

6. Sun Q, Xiao X, Kim Y, Kim D, Yoon KS, Clark JM, et al. Imidacloprid Promotes High Fat Diet-Induced Adiposity and Insulin Resistance in Male C57BL/6J Mice. Journal of Agricultural and Food Chemistry. 2016;64(49):9293-306; doi: 10.1021/acs.jafc.6b04322.

7. Xiao X, Sun Q, Kim Y, Yang S-H, Qi W, Kim D, et al. Exposure to permethrin promotes high fat dietinduced weight gain and insulin resistance in male C57BL/6J mice. Food and Chemical Toxicology. 2018;111:405-16; doi: 10.1016/j.fct.2017.11.047.

8. Mostafalou S, Abdollahi M. Pesticides: an update of human exposure and toxicity. Archives of Toxicology. 2017;91(2):549-99; doi: 10.1007/s00204-016-1849-x.

9. Lee D-H, Lind L, Jacobs DR, Jr., Salihovic S, van Bavel B, Lind PM. Associations of persistent organic pollutants with abdominal obesity in the elderly: The Prospective Investigation of the Vasculature in Uppsala Seniors (PIVUS) study. Environment International. 2012;40:170-8; doi:

10.1016/j.envint.2011.07.010.

10. Santovito A, Gendusa C, Ferraro F, Musso I, Costanzo M, Ruberto S, et al. Genomic damage induced by the widely used fungicide chlorothalonil in peripheral human lymphocytes. Ecotoxicology and Environmental Safety. 2018;161:578-83; doi: 10.1016/j.ecoenv.2018.06.047.

11. Zhang H, Nie Y, Zhang S, Wang WZ, Li H, Wang F, et al. Monitoring and probabilistic risk assessment of chlorothalonil residues in vegetables from Shandong province (China). Regulatory Toxicology and Pharmacology. 2016;80:41-5; doi: 10.1016/j.yrtph.2016.05.035. 
12. Wanwimolruk S, Kanchanamayoon O, Phopin K, Prachayasittikul V. Food safety in Thailand 2: Pesticide residues found in Chinese kale (Brassica oleracea), a commonly consumed vegetable in Asian countries. Science of the Total Environment. 2015;532:447-55; doi: 10.1016/j.scitotenv.2015.04.114.

13. Budai P, Szabo R, Lehel J, Kormos E, Takacs A, Tatai A, et al. Toxicity of chlorothalonil containing formulation and $\mathrm{Cu}$-sulphate to chicken. Communications in agricultural and applied biological sciences. 2012;77(4):449-54.

14. Du Gas LM, Ross PS, Walker J, Marlatt VL, Kennedy CJ. Effects of atrazine and chlorothalonil on the reproductive success, development, and growth of early life stage sockeye salmon (oncorhynchus nerka). Environmental Toxicology and Chemistry. 2017;36(5):1354-64; doi: 10.1002/etc.3753.

15. Onduka T, Kakuno A, Kono K, Ito K, Mochida K, Fujii K. Toxicity of chlorothalonil to marine organisms. Fisheries Science. 2012;78(6):1301-8; doi: 10.1007/s12562-012-0562-9.

16. Salis S, Testa C, Roncada P, Armorini S, Rubattu N, Ferrari A, et al. Occurrence of imidacloprid, carbendazim, and other biocides in Italian house dust: Potential relevance for intakes in children and pets. Journal of Environmental Science and Health Part B-Pesticides Food Contaminants and Agricultural Wastes. 2017;52(9):699-709; doi: 10.1080/03601234.2017.1331675.

17. Zhang P, Zhao Y, Zhang H, Liu J, Feng Y, Yin S, et al. Low dose chlorothalonil impairs mouse spermatogenesis through the intertwining of Estrogen Receptor Pathways with histone and DNA methylation. Chemosphere. 2019;230:384-95; doi: 10.1016/j.chemosphere.2019.05.029.

18. Garayzar ABS, Bahamonde PA, Martyniuk CJ, Betancourt M, Munkittrick KR. Hepatic gene expression profiling in zebrafish (Danio rerio) exposed to the fungicide chlorothalonil. Comparative Biochemistry and Physiology D-Genomics \& Proteomics. 2016;19:102-11; doi: 10.1016/j.cbd.2016.04.004.

19. Yu S, Wages MR, Cobb GP, Maul JD. Effects of chlorothalonil on development and growth of amphibian embryos and larvae. Environmental Pollution. 2013;181:329-34; doi:

10.1016/j.envpol.2013.06.017.

20. Hao Y, Zhang H, Zhang P, Yu S, Ma D, Li L, et al. Chlorothalonil inhibits mouse ovarian development through endocrine disruption. Toxicology Letters. 2019;303:38-47; doi: 10.1016/j.toxlet.2018.12.011.

21. Wang Y, Jin C, Wang D, Zhou J, Yang G, Shao K, et al. Effects of chlorothalonil, prochloraz and the combination on intestinal barrier function and glucolipid metabolism in the liver of mice. Journal of Hazardous Materials. 2021;410; doi: 10.1016/j.jhazmat.2020.124639.

22. Huttenhower $\mathrm{C}$, Gevers D, Knight R, Abubucker S, Badger JH, Chinwalla AT, et al. Structure, function and diversity of the healthy human microbiome. Nature. 2012;486(7402):207-14; doi:

10.1038/nature11234. 
23. Sanz Y, Olivares M, Moya-Perez A, Agostoni C. Understanding the role of gut microbiome in metabolic disease risk. Pediatric Research. 2015;77(1):236-44; doi: 10.1038/pr.2014.170.

24. Clemente JC, Ursell LK, Parfrey LW, Knight R. The Impact of the Gut Microbiota on Human Health: An Integrative View. Cell. 2012;148(6):1258-70; doi: 10.1016/j.cell.2012.01.035.

25. Louis P, Hold GL, Flint HJ. The gut microbiota, bacterial metabolites and colorectal cancer. Nature Reviews Microbiology. 2014;12(10):661-72; doi: 10.1038/nrmicro3344.

26. Blacher E, Levy M, Tatirovsky E, Elinav E. Microbiome-Modulated Metabolites at the Interface of Host Immunity. Journal of Immunology. 2017;198(2):572-80; doi: 10.4049/jimmunol.1601247.

27. Agus A, Planchais J, Sokol H. Gut Microbiota Regulation of Tryptophan Metabolism in Health and Disease. Cell Host \& Microbe. 2018;23(6):716-24; doi: 10.1016/j.chom.2018.05.003.

28. Vallim TQdA, Tarling EJ, Edwards PA. Pleiotropic Roles of Bile Acids in Metabolism. Cell Metabolism. 2013;17(5):657-69; doi: 10.1016/j.cmet.2013.03.013.

29. Wahlstrom A, Sayin SI, Marschall H-U, Backhed F. Intestinal Crosstalk between Bile Acids and Microbiota and Its Impact on Host Metabolism. Cell Metabolism. 2016;24(1):41-50; doi:

10.1016/j.cmet.2016.05.005.

30. Schuster S, Cabrera D, Arrese M, Feldstein AE. Triggering and resolution of inflammation in NASH. Nature Reviews Gastroenterology \& Hepatology. 2018;15(6):349-64; doi: 10.1038/s41575-018-0009-6.

31. Friedman SL, Neuschwander-Tetri BA, Rinella M, Sanyal AJ. Mechanisms of NAFLD development and therapeutic strategies. Nature Medicine. 2018;24(7):908-22; doi: 10.1038/s41591-018-0104-9.

32. Watanabe M, Houten SM, Wang L, Moschetta A, Mangelsdorf DJ, Heyman RA, et al. Bile acids lower triglyceride levels via a pathway involving FXR, SHP, and SREBP-1c. Journal of Clinical Investigation. 2004;113(10):1408-18; doi: 10.1172/jci200421025.

33. Zhang YQ, Lee FY, Barrera G, Lee H, Vales C, Gonzalez FJ, et al. Activation of the nuclear receptor FXR improves hyperglycemia and hyperlipidemia in diabetic mice. Proceedings of the National Academy of Sciences of the United States of America. 2006;103(4):1006-11; doi: 10.1073/pnas.0506982103.

34. Parseus A, Sommer N, Sommer F, Caesar R, Molinaro A, Stahlman M, et al. Microbiota-induced obesity requires farnesoid X receptor. Gut. 2017;66(3):429-37; doi: 10.1136/gutjnl-2015-310283.

35. Li F, Jiang C, Krausz KW, Li Y, Albert I, Hao H, et al. Microbiome remodelling leads to inhibition of intestinal farnesoid $X$ receptor signalling and decreased obesity. Nature Communications. 2013;4; doi: $10.1038 /$ ncomms3384. 
36. Yuan X, Pan Z, Jin C, Ni Y, Fu Z, Jin Y. Gut microbiota: An underestimated and unintended recipient for pesticide-induced toxicity. Chemosphere. 2019;227:425-34; doi: 10.1016/j.chemosphere.2019.04.088.

37. Meng Z, Liu L, Yan S, Sun W, Jia M, Tian S, et al. Gut Microbiota: A Key Factor in the Host Health Effects Induced by Pesticide Exposure? Journal of Agricultural and Food Chemistry. 2020;68(39):1051731; doi: 10.1021/acs.jafc.0c04678.

38. Liang YR, Zhan J, Liu DH, Luo M, Han JJ, Liu XK, et al. Organophosphorus pesticide chlorpyrifos intake promotes obesity and insulin resistance through impacting gut and gut microbiota. Microbiome. 2019;7; doi: 10.1186/s40168-019-0635-4.

39. A YW, B CJ, A DW, B JZ, A GY, C KS, et al. Effects of chlorothalonil, prochloraz and the combination on intestinal barrier function and glucolipid metabolism in the liver of mice - ScienceDirect. Journal of Hazardous Materials. 2020.

40. Lim MY, You HJ, Yoon HS, Kwon B, Lee JY, Lee S, et al. The effect of heritability and host genetics on the gut microbiota and metabolic syndrome. Gut. 2017;66(6):1031-8; doi: 10.1136/gutjnl-2015-311326.

41. Dabke K, Hendrick G, Devkota S. The gut microbiome and metabolic syndrome. Journal of Clinical Investigation. 2019;129(10):4050-7; doi: 10.1172/jci129194.

42. Natividad JM, Agus A, Planchais J, Lamas B, Jarry AC, Martin R, et al. Impaired Aryl Hydrocarbon Receptor Ligand Production by the Gut Microbiota Is a Key Factor in Metabolic Syndrome. Cell Metabolism. 2018;28(5):737-+; doi: 10.1016/j.cmet.2018.07.001.

43. Krautkramer KA, Fan J, Backhed F. Gut microbial metabolites as multi-kingdom intermediates. Nature Reviews Microbiology. 2021;19(2):77-94; doi: 10.1038/s41579-020-0438-4.

44. Yueh M-F, He F, Chen C, Vu C, Tripathi A, Knight R, et al. regulator FGF21 exacerbating high fat dietinduced nonalcoholic fatty liver disease. Proceedings of the National Academy of Sciences of the United States of America. 2020;117(49):31259-66; doi: 10.1073/pnas.2017129117.

45. Kaliannan K, Robertson RC, Murphy K, Stanton C, Kang C, Wang B, et al. Estrogen-mediated gut microbiome alterations influence sexual dimorphism in metabolic syndrome in mice. Microbiome. 2018;6; doi: 10.1186/s40168-018-0587-0.

46. Jin Y, Wu S, Zeng Z, Fu Z. Effects of environmental pollutants on gut microbiota. Environmental Pollution. 2017;222:1-9; doi: 10.1016/j.envpol.2016.11.045.

47. Chassaing B, Koren O, Goodrich JK, Poole AC, Srinivasan S, Ley RE, et al. Dietary emulsifiers impact the mouse gut microbiota promoting colitis and metabolic syndrome. Nature. 2015;519(7541):92-U192; doi: 10.1038/nature14232. 
48. Turnbaugh PJ, Baeckhed F, Fulton L, Gordon JI. Diet-induced obesity is linked to marked but reversible alterations in the mouse distal gut microbiome. Cell Host \& Microbe. 2008;3(4):213-23; doi: 10.1016/j.chom.2008.02.015.

49. Ley RE, Turnbaugh PJ, Klein S, Gordon JI. Microbial ecology - Human gut microbes associated with obesity. Nature. 2006;444(7122):1022-3; doi: 10.1038/4441022a.

50. Ley RE, Backhed F, Turnbaugh P, Lozupone CA, Knight RD, Gordon JI. Obesity alters gut microbial ecology. Proceedings of the National Academy of Sciences of the United States of America. 2005;102(31):11070-5; doi: 10.1073/pnas.0504978102.

51. Duca FA, Sakar Y, Lepage P, Devime F, Langelier B, Dore J, et al. RETRACTION: Replication of Obesity and Associated Signaling Pathways Through Transfer of Microbiota From Obese-Prone Rats. Diabetes (Retraction of vol 63, pg 1624, 2014). Diabetes. 2016;65(5):1447-; doi: 10.2337/db16-rt05.

52. Ormerod KL, Wood DLA, Lachner N, Gellatly SL, Daly JN, Parsons JD, et al. Genomic characterization of the uncultured Bacteroidales family S24-7 inhabiting the guts of homeothermic animals. Microbiome. 2016;4; doi: 10.1186/s40168-016-0181-2.

53. Liu R, Hong J, Xu X, Feng Q, Zhang D, Gu Y, et al. Gut microbiome and serum metabolome alterations in obesity and after weight-loss intervention. Nature Medicine. 2017;23(7):859-+; doi: 10.1038/nm.4358.

54. Delzenne NM, Bindels LB. Microbiome metabolomics reveals new drivers of human liver steatosis. Nature Medicine. 2018;24(7):906-7; doi: 10.1038/s41591-018-0126-3.

55. Nicholson JK, Holmes E, Kinross J, Burcelin R, Gibson G, Jia W, et al. Host-Gut Microbiota Metabolic Interactions. Science. 2012;336(6086):1262-7; doi: 10.1126/science.1223813.

56. Ma C, Han MJ, Heinrich B, Fu Q, Zhang QF, Sandhu M, et al. Gut microbiome-mediated bile acid metabolism regulates liver cancer via NKT cells. Science. 2018;360(6391); doi:

10.1126/science.aan5931.

57. Zhao L, Yang W, Chen Y, Huang FJ, Lu L, Lin CY, et al. A Clostridia-rich microbiota enhances bile acid excretion in diarrhea-predominant irritable bowel syndrome. Journal of Clinical Investigation. 2020;130(1):438-50; doi: 10.1172/jci130976.

58. Guo JL, Han X, Tan HY, Huang WD, You YL, Zhan JC. Blueberry Extract Improves Obesity through Regulation of the Gut Microbiota and Bile Acids via Pathways Involving FXR and TGR5. Iscience. 2019;19:676-+; doi: 10.1016/j.isci.2019.08.020.

59. Chen L, van den Munckhof IC, Schraa K, ter Horst R, Koehorst M, van Faassen M, et al. Genetic and Microbial Associations to Plasma and Fecal Bile Acids in Obesity Relate to Plasma Lipids and Liver Fat Content. Cell Reports. 2020;33(1); doi: 10.1016/j.celrep.2020.108212. 
60. Huang F, Zheng X, Ma X, Jiang R, Zhou W, Zhou S, et al. Theabrownin from Pu-erh tea attenuates hypercholesterolemia via modulation of gut microbiota and bile acid metabolism. Nature Communications. 2019;10; doi: 10.1038/s41467-019-12896-x.

61. Wang HB, Chen J, Hollister K, Sowers LC, Forman BM. Endogenous bile acids are ligands for the nuclear receptor FXR BAR. Molecular Cell. 1999;3(5):543-53; doi: 10.1016/s1097-2765(00)80348-2.

62. Sayin SI, Wahlstrom A, Felin J, Jantti S, Marschall H-U, Bamberg K, et al. Gut Microbiota Regulates Bile Acid Metabolism by Reducing the Levels of Tauro-beta-muricholic Acid, a Naturally Occurring FXR Antagonist. Cell Metabolism. 2013;17(2):225-35; doi: 10.1016/j.cmet.2013.01.003.

63. Schmitt J, Kong B, Stieger B, Tschopp O, Schultze SM, Rau M, et al. Protective effects of farnesoid X receptor (FXR) on hepatic lipid accumulation are mediated by hepatic FXR and independent of intestinal FGF15 signal. Liver International. 2015;35(4):1133-44; doi: 10.1111/liv.12456.

\section{Supplementary Files}

This is a list of supplementary files associated with this preprint. Click to download.

- 20211017Supportinginformation.docx 